\title{
CONTRACTIBILITY OF THE STABILITY MANIFOLD FOR SILTING-DISCRETE ALGEBRAS
}

\author{
DAVID PAUKSZTELLO, MANUEL SAORÍN, AND ALEXANDRA ZVONAREVA
}

\begin{abstract}
We show that any bounded t-structure in the bounded derived category of a silting-discrete algebra is algebraic, i.e. has a length heart with finitely many simple objects. As a corollary, we obtain that the space of Bridgeland stability conditions for a silting-discrete algebra is contractible.
\end{abstract}

\section{INTRODUCTION}

Stability conditions on triangulated categories were introduced by Bridgeland in [12] as a means of extracting geometry from homological algebra with a view to constructing moduli spaces arising in the context of Homological Mirror Symmetry. They can be thought of as a continuous generalisation of bounded t-structures. The main result of [12] asserts that the space of stability conditions forms a complex manifold, the stability manifold. This can be thought of as geometrically encoding most of the cohomology theories on a given triangulated category.

Bounded t-structures admit a mutation theory given by HRS-tilts (see Proposition 2.3 below), giving rise to a graph that is closely related to the exchange graphs occurring in cluster combinatorics [24, which is the skeleton of the stability manifold in the Dynkin case. Despite being the focus of extensive investigation, for example [12, 14, 17, 18, 20, 26, 28, 31, 32], computations with stability conditions are difficult. For example, it is widely believed that whenever the stability manifold is nonempty it is contractible. However, this has been proved in only few cases, though the list is now growing, see [14, 18, 20, 26, 28, 32].

Silting objects are a generalisation of tilting objects due to Keller and Vossieck in [23]. In the context of bounded derived categories of finite-dimensional algebras, silting objects enable the detection of t-structures whose hearts are equivalent to module categories of finite-dimensional algebras [25]. Silting-discreteness [4] is a finiteness condition on a triangulated category that says there are only finitely many silting objects in any interval in the poset of silting objects [5]; see below for precise definitions. Examples of siltingdiscrete finite-dimensional algebras include hereditary algebras of finite representation type, derived-discrete algebras [14], preprojective algebras of Dynkin type [6], symmetric algebras of finite representation type [4], Brauer graph algebras whose Brauer graphs contain at most one cycle of odd length and no cycles of even length [1], and local algebras [5].

The purpose of this paper is to establish the following property of the bounded tstructures in the bounded derived category $\mathrm{D}^{b}(\Lambda)$ of a silting-discrete finite-dimensional

2010 Mathematics Subject Classification. 18E30, 16G10.

Key words and phrases. Bounded t-structure, silting-discrete, stability condition.

Alexandra Zvonareva is supported by the RFBR Grant 16-31-60089. Manuel Saorín is supported by research projects from the Ministerio de Economía y Competitividad of Spain (MTM2016-77445P) and from the Fundación 'Séneca' of Murcia (19880/GERM/15), both with a part of FEDER funds. 
algebra $\Lambda$. We recall that a bounded t-structure is algebraic if it is given by a silting object; see Section 2 for the precise definition.

Theorem A. If $\Lambda$ is a silting-discrete finite-dimensional $\mathbf{k}$-algebra, then any bounded t-structure in $\mathrm{D}^{b}(\Lambda)$ is algebraic, i.e. has a length heart.

In particular, this result means that any bounded t-structure $(X, Y)$ in $D^{b}(\Lambda)$ admits only finitely many HRS tilts. Hence, the techniques and methods used in [14, 32] to show that the stability manifold of a derived-discrete algebra is contractible can be applied here.

Corollary B. If $\Lambda$ is a silting-discrete finite-dimensional $\mathbf{k}$-algebra, then the stability manifold $\operatorname{stab}\left(\mathrm{D}^{b}(\Lambda)\right)$ is contractible.

In [3, Adachi, Mizuno and Yang have independently obtained similar results in the setting of silting-discrete triangulated categories.

The outline of this paper is as follows. In Section 2 we recall the concepts and results that will be necessary to establish Theorem A. In Section 3 we prove Theorem A. Once one has Theorem $\mathrm{A}$ the proof of Corollary $\mathrm{B}$ is implicit in [14, 32. For the convenience of the reader we briefly sketch the narrative of the argument in [14, 32] in Section 4.

Convention. Throughout this paper all subcategories are full and strict, $\mathbf{k}$ is a field, and all algebras are finite-dimensional $\mathbf{k}$-algebras. For a finite-dimensional $\mathbf{k}$-algebra $\Lambda$ we denote by $\bmod (\Lambda)$ the category of finite-dimensional right $\Lambda$-modules; by $\mathrm{K}^{b}(\operatorname{proj}(\Lambda))$ we denote the bounded homotopy category of complexes of finite-dimensional projective $\Lambda$-modules; by $\mathrm{D}^{b}(\Lambda)$ we denote the bounded derived category of finite-dimensional right $\Lambda$-modules; and by $\mathrm{D}(\Lambda)$ we denote the unbounded derived category of right $\Lambda$-modules. Throughout $\mathrm{D}$ is a triangulated category and the shift functor is denoted by $[1]: \mathrm{D} \rightarrow \mathrm{D}$.

\section{BACKGROUND}

For a subcategory $\mathrm{S}$ of a triangulated category $\mathrm{D}$ we define

$$
\mathrm{S}[>n]=\{S[i] \mid S \in \mathrm{S}, i>n\} \text { and } \mathrm{S}[<n]=\{S[i] \mid S \in \mathrm{S}, i<n\},
$$

analogously for $\mathrm{S}[\leq n]$ and $\mathrm{S}[\geq n]$. For subcategories $\mathrm{X}$ and $\mathrm{Y}$ of $\mathrm{D}$ we define $\mathrm{X} * \mathrm{Y}=\{D \in \mathrm{D} \mid$ there exists a triangle $X \rightarrow D \rightarrow Y \rightarrow X[1]$ with $X \in \mathrm{X}$ and $Y \in \mathrm{Y}\}$.

A subcategory $\mathrm{X}$ is extension closed if $\mathrm{X}=\mathrm{X} * \mathrm{X}$. We shall denote the extension closure of $\mathrm{X}$ by $\langle\mathbf{X}\rangle$. We define the right and left perpendicular categories of $\mathrm{X}$ respectively by

$$
\begin{aligned}
& \mathrm{X}^{\perp}=\left\{D \in \mathrm{D} \mid \operatorname{Hom}_{\mathrm{D}}(X, D)=0 \text { for all } X \in \mathrm{X}\right\} \\
& { }^{\perp} \mathrm{X}=\left\{D \in \mathrm{D} \mid \operatorname{Hom}_{\mathrm{D}}(D, X)=0 \text { for all } X \in \mathrm{X}\right\} .
\end{aligned}
$$

For subcategories of an abelian category $\mathrm{H}$ we use the same notation for the analogous definitions, using short exact sequences instead of triangles.

2.1. Torsion pairs and t-structures. The general notion of a torsion pair on an abelian category goes back to [16].

Definition 2.1. A torsion pair in an abelian category $\mathrm{H}$ consists of a pair of full subcategories $(\mathcal{T}, \mathcal{F})$ such that $\mathcal{T}^{\perp}=\mathcal{F}, \mathcal{T}={ }^{\perp} \mathcal{F}$, and $\mathrm{H}=\mathcal{T} * \mathcal{F}$. We call $\mathcal{T}$ the torsion class and $\mathcal{F}$ the torsionfree class of the torsion pair. 
If the abelian category $\mathrm{H}$ is $\bmod (\Lambda)$ for a finite-dimensional algebra $\Lambda$, then any subcategory $\mathcal{T}$ closed under extensions, factor objects and direct summands gives rise to a torsion class of a torsion pair; see, e.g. [8, Ch. VI]. A dual statement holds for torsionfree classes. For $M \in \bmod (\Lambda)$ we write $\operatorname{Fac}(M)$ for the full subcategory of all factor modules of finite direct sums of copies of $M$. When $M$ is a support $\tau$-tilting module (see Section 2.2 below), $\operatorname{Fac}(M)$ is the smallest torsion class containing $M$.

The analogue of a torsion pair in a triangulated category is a t-structure [10].

Definition 2.2. A t-structure on a triangulated category $D$ consists of a pair of full subcategories $(\mathrm{X}, \mathrm{Y})$ such that $\mathrm{X}^{\perp}=\mathrm{Y}, \mathrm{X}={ }^{\perp} \mathrm{Y}, \mathrm{D}=\mathrm{X} * \mathrm{Y}$ and $\mathrm{X}[1] \subseteq \mathrm{X}$ (equivalently, $\mathrm{Y}[-1] \subseteq \mathrm{Y})$. The subcategory $\mathrm{H}=\mathrm{X} \cap \mathrm{Y}[1]$ is an abelian subcategory of $\mathrm{D}$ called the heart of $(\mathrm{X}, \mathrm{Y})$. A t-structure is called bounded if

$$
\mathrm{D}=\bigcup_{n \in \mathbb{Z}} \mathbf{X}[n]=\bigcup_{n \in \mathbb{Z}} \mathrm{Y}[n] .
$$

For a bounded t-structure $(X, Y)$ we have $X=\langle H[\geq 0]\rangle$ and $Y=\langle H[<0]\rangle$. A t-structure is called algebraic if it is bounded and $\mathrm{H}$ is a length category, i.e. $\mathrm{H}$ has finitely many isomorphism classes of simple objects and each object of $\mathrm{H}$ is both Artinian and Noetherian.

There is a close connection between torsion pairs and t-structures.

Proposition 2.3 ([11, 29, 34]). Suppose $(\mathrm{X}, \mathrm{Y})$ is a t-structure on $\mathrm{D}$ with heart $\mathrm{H}$. Then there is a bijection

$$
\begin{aligned}
\text { t-structures } \left.\left(\mathrm{X}^{\prime}, \mathrm{Y}^{\prime}\right) \text { with } \mathrm{X}[1] \subseteq \mathrm{X}^{\prime} \subseteq \mathrm{X}\right\} & \stackrel{1-1}{\longleftrightarrow} \text { \{torsion pairs }(\mathcal{T}, \mathcal{F}) \text { in } \mathrm{H}\} \\
\left(\mathrm{X}^{\prime}, \mathrm{Y}^{\prime}\right) & \longleftrightarrow\left(\mathcal{T}=\mathrm{H} \cap \mathrm{X}^{\prime}, \mathcal{F}=\mathrm{H} \cap \mathrm{Y}^{\prime}\right) ; \\
\left(\mathrm{X}^{\prime}=\langle\mathcal{T}, \mathrm{X}[1]\rangle, \mathrm{Y}^{\prime}=\langle\mathrm{Y}, \mathcal{F}\rangle\right) & \longleftrightarrow(\mathcal{T}, \mathcal{F}) .
\end{aligned}
$$

The t-structure $\left(\mathrm{X}^{\prime}, \mathrm{Y}^{\prime}\right)$ in Proposition 2.3 is called a left $H R S$-tilt of $(\mathrm{X}, \mathrm{Y})$ at the torsion pair $(\mathcal{T}, \mathcal{F})$ and is called intermediate with respect to $(\mathrm{X}, \mathrm{Y})$; see [21, Proposition I.2.1]. Note that $\mathrm{X}^{\prime}=\mathrm{X}[1] * \mathcal{T}$ and $Y^{\prime}=\mathcal{F} * \mathrm{Y}$.

2.2. Silting, t-structures and $\tau$-tilting. Silting was first introduced in [23]; however, we follow the treatment of [5].

Definition 2.4. A subcategory $\mathrm{S}$ of $\mathrm{D}$ is silting if thick $(\mathrm{S})=\mathrm{D}$ and $\operatorname{Hom}_{\mathrm{D}}\left(S, S^{\prime}[i]\right)=0$ for each $S, S^{\prime} \in \mathrm{S}$ and $i>0$, where thick(S) is the smallest triangulated subcategory of $\mathrm{D}$ containing $\mathrm{S}$ that is closed under direct summands. An object $S$ of $\mathrm{D}$ is a silting object if $\operatorname{add}(S)$ is a silting subcategory, where add $(S)$ consists of the direct summands of finite coproducts of copies of $S$.

For a finite-dimensional algebra $\Lambda$ we shall freely abuse notation and identify silting subcategories with silting objects, since any silting subcategory in $\mathrm{K}^{b}(\operatorname{proj}(\Lambda))$ is of the form $\operatorname{add}(S)$, for some silting object uniquely determined up to additive closure.

There is a partial order on silting subcategories of D (see [5]): for silting subcategories $\mathrm{S}$ and $\mathrm{T}$,

$$
\mathrm{S} \geq \mathrm{T} \text { if and only if } \operatorname{Hom}_{\mathrm{D}}(S, T[i])=0 \text { for all } S \in \mathrm{S}, T \in \mathrm{T} \text { and } i>0,
$$

which is equivalent to $\mathrm{T} \subseteq(\mathrm{S}[<0])^{\perp}$. We denote this poset by $\mathbb{P}_{1}(\mathrm{D})$. A silting subcategory $\mathrm{T}$ is called two term with respect to $\mathrm{S}$ if $\mathrm{S} \geq \mathrm{T} \geq \mathrm{S}[1]$, which happens if and only if $\mathrm{T} \in \mathrm{S} * \mathrm{~S}[1]$; see, for example, [22]. 
Definition 2.5 ([4, Definition 3.6 \& Proposition 3.8]). A finite-dimensional algebra $\Lambda$ is silting-discrete if for any silting object $S$ and any natural number $n$ there are only finitely many silting objects $T$ such that $S \geq T \geq S[n]$. Note that, via [32, Lemma 2.14], this is equivalent to there being only finitely many silting objects $T$ such that $S \geq T \geq S[1]$.

In the case that $\mathrm{D}=\mathrm{D}^{b}(\Lambda)$ for a finite-dimensional algebra $\Lambda$, there is a correspondence between silting subcategories and algebraic t-structures.

Theorem 2.6 ([25] \& [22]). Let $\Lambda$ be a finite-dimensional $\mathbf{k}$-algebra. Then there is a bijection

$$
\begin{aligned}
\left\{\text { silting subcategories of } \mathrm{K}^{b}(\operatorname{proj}(\Lambda))\right\} & \left.\stackrel{1-1}{\longleftrightarrow} \text { \{algebraic t-structures on } \mathrm{D}^{b}(\Lambda)\right\} ; \\
\mathrm{S} & \longmapsto\left(\mathrm{X}_{\mathrm{S}}=(\mathrm{S}[<0])^{\perp}, \mathrm{Y}_{\mathrm{S}}=(\mathrm{S}[\geq 0])^{\perp}\right)
\end{aligned}
$$

Moreover, this restricts to a bijection with intermediate algebraic t-structures,

$\{$ silting subcategories $\mathrm{T} \subseteq \mathrm{S} * \mathrm{~S}[1]\} \stackrel{1-1}{\longleftrightarrow}\left\{\right.$ algebraic t-structures $(\mathrm{X}, \mathrm{Y})$ with $\left.\mathrm{X}_{\mathrm{S}}[1] \subseteq \mathrm{X} \subseteq \mathrm{X}_{\mathrm{S}}\right\}$.

Definition 2.7. Let $\Lambda$ be a finite-dimensional k-algebra and denote the Auslander-Reiten translation on $\bmod (\Lambda)$ by $\tau$. Write $|M|$ for the number of nonisomorphic indecomposable summands of a $\Lambda$-module $M$.

(1) ([2, Definitions $0.1 \& 0.3])$ A pair $(M, P) \in \bmod (\Lambda) \times \operatorname{proj}(\Lambda)$ is a $\tau$-rigid pair if $\operatorname{Hom}_{\Lambda}(M, \tau M)=0$ and $\operatorname{Hom}_{\Lambda}(P, M)=0$. A $\tau$-rigid pair is a support $\tau$-tilting pair if $|M|+|P|=|\Lambda|$. In this case $M$ is called a support $\tau$-tilting module. If in a support $\tau$-tilting pair $P=0$, we call $M$ a $\tau$-tilting module.

(2) ([15, Definition 1.1]) The algebra $\Lambda$ is $\tau$-tilting finite if there are only finitely many isomorphism classes of basic $\tau$-tilting $\Lambda$-modules.

The following characterisation of support $\tau$-tilting pairs will be useful.

Lemma 2.8 ([2, Corollary 2.13], see also [7, Theorem 2.5(3)]). Let $M \in \bmod (\Lambda)$ and

$$
P_{1} \stackrel{\sigma}{\rightarrow} P_{0} \rightarrow M \rightarrow 0
$$

be its minimal projective presentation. The pair $(M, P)$ is support $\tau$-tilting if and only if $\operatorname{Fac}(M)$ consists of the $N \in \bmod (\Lambda)$ such that $\operatorname{Hom}_{\Lambda}(\widetilde{\sigma}, N)$ is surjective, where $\widetilde{\sigma}=\left[\begin{array}{ll}\sigma & 0\end{array}\right]$ in the projective presentation

$$
P_{1} \oplus P \stackrel{\widetilde{\sigma}}{\rightarrow} P_{0} \rightarrow M
$$

A result of 15 relates $\tau$-tilting finiteness with functorial finiteness of torsion classes; we refer the reader to, for example [8], for the definition of functorial finiteness.

Theorem 2.9 ([15, Theorem 3.8]). A finite-dimensional algebra $\Lambda$ is $\tau$-tilting finite if and only if every torsion class (equivalently, every torsionfree class) in $\bmod (\Lambda)$ is functorially finite.

The results of [2] combined with [22] give the following theorem.

Theorem 2.10 ([22, Theorem 4.6] and [2]). Let D be a Krull-Schmidt, Hom-finite, klinear triangulated category and let $\mathrm{S}=\operatorname{add}(S)$ for a silting object $S$. Let $\Gamma=\operatorname{End}_{\mathrm{D}}(S)$. Then there is a bijection between the following sets.

(1) Basic silting objects $T$ of $\mathrm{D}$ with $T \in S * S[1]$, modulo isomorphism.

(2) Basic support $\tau$-tilting pairs of $\bmod (\Gamma)$, modulo isomorphism.

(3) Torsion pairs $(\mathcal{T}, \mathcal{F})$ in $\bmod (\Gamma)$ in which $\mathcal{T}$ and $\mathcal{F}$ are functorially finite. 
Remark 2.11. Suppose we are in the setup of Theorem 2.10. Recall from [22, Remark 4.1(ii)] that there is an equivalence $\operatorname{Mod}(S) \simeq \operatorname{Mod}(\Gamma)$, where $\operatorname{Mod}(S)$ is the category of contravariant functors from $\mathrm{S}$ to the category of abelian groups. Let $(M, P)$ be a support $\tau$-tilting pair of $\bmod (\Gamma)$ with minimal projective presentation

$$
P_{1} \stackrel{\sigma}{\rightarrow} P_{0} \rightarrow M \rightarrow 0
$$

and the 'extended' presentation

$$
\widetilde{P}=P_{1} \oplus P \stackrel{\widetilde{\sigma}}{\rightarrow} P_{0} \rightarrow M \rightarrow 0
$$

of Lemma 2.8. One can uniquely lift this presentation to $\operatorname{Mod}(\mathrm{S})$ as

$$
\left.\left.\operatorname{Hom}_{\mathrm{D}}(-, \widetilde{S})\right|_{\mathrm{S}} \stackrel{(-, f)}{\rightarrow} \operatorname{Hom}_{\mathrm{D}}\left(-, S_{0}\right)\right|_{\mathrm{S}}
$$

where $\left.\operatorname{Hom}_{\mathrm{D}}(-, D)\right|_{\mathrm{S}}$ denotes the image of $D$ under the restricted Yoneda functor [9]; cf. [22, Remark 3.1]. The corresponding silting object $T \in \mathrm{S} * \mathrm{~S}[1]$ is then the mapping cone of $f: \widetilde{S} \rightarrow S_{0}$ in D.

\section{Proof of Theorem A}

We start by showing that when $\Lambda$ is silting-discrete any HRS-tilt of an algebraic tstructure is again algebraic.

Proposition 3.1. Let $\Lambda$ be a silting-discrete finite-dimensional algebra. Let $\mathrm{S} \subseteq \mathrm{K}^{b}(\operatorname{proj}(\Lambda))$ be a silting subcategory and let $\left(\mathrm{X}_{\mathrm{S}}, \mathrm{Y}_{\mathrm{S}}\right)$ be the corresponding algebraic t-structure on $\mathrm{D}^{b}(\Lambda)$. If $(\mathrm{X}, \mathrm{Y})$ is a t-structure intermediate with respect to $\left(\mathrm{X}_{\mathrm{S}}, \mathrm{Y}_{\mathrm{S}}\right)$ then $(\mathrm{X}, \mathrm{Y})$ is algebraic.

Proof. Suppose $(\mathrm{X}, \mathrm{Y})$ is a t-structure intermediate with respect to an algebraic t-structure $\left(\mathrm{X}_{\mathrm{S}}, \mathrm{Y}_{\mathrm{S}}\right)$, where $\mathrm{S}=\operatorname{add}(S)$ for some basic silting object $S$. First observe that since $(\mathrm{X}, \mathrm{Y})$ is intermediate with respect to a bounded t-structure $\left(X_{S}, Y_{S}\right)$ it is automatically bounded. Let $\Gamma=\operatorname{End}_{\mathrm{K}^{b}(\operatorname{proj}(\Lambda))}(S)$ and note that $\mathrm{H}_{\mathrm{S}} \simeq \bmod (\Gamma)$ by [25]. Since $\Lambda$ is silting-discrete, there are finitely many silting objects in $\mathrm{S} * \mathrm{~S}[1]$, and therefore, by Theorem 2.10, finitely many support $\tau$-tilting pairs in $\bmod (\Gamma)$, whence $\Gamma$ is $\tau$-tilting finite.

By Proposition 2.3, there exists a torsion pair $(\mathcal{T}, \mathcal{F})$ on $\mathrm{H}_{\mathrm{S}}$ such that $\mathrm{X}=\left\langle\mathcal{T}, \mathrm{X}_{\mathrm{S}}[1]\right\rangle$ and $\mathrm{Y}=\left\langle\mathrm{Y}_{\mathrm{S}}, \mathcal{F}\right\rangle$. By Theorem 2.9. $\mathcal{T}$ and $\mathcal{F}$ are functorially finite, so that by the correspondence in Theorem 2.10, $\mathcal{T}=\operatorname{Fac}(M)$ for some support $\tau$-tilting pair $(M, P)$ of $\bmod (\Gamma)$, which in turn corresponds to some silting object $T \in \mathrm{S} * \mathrm{~S}[1]$. By Theorem 2.6, this corresponds to an algebraic t-structure $\left(\mathrm{X}_{\mathrm{T}}, \mathrm{Y}_{\mathrm{T}}\right)$ that is intermediate with respect to $\left(\mathrm{X}_{\mathrm{S}}, \mathrm{Y}_{\mathrm{S}}\right)$. Invoking Proposition 2.3 again, there is a torsion pair $\left(\mathcal{T}_{\mathrm{T}}, \mathcal{F}_{\mathrm{T}}\right)$ on $\mathrm{H}_{\mathrm{S}}$ such that $\mathrm{X}_{\mathrm{T}}=\left\langle\mathcal{T}_{\mathrm{T}}, \mathrm{X}_{\mathrm{S}}[1]\right\rangle$ and $\mathrm{Y}_{\mathrm{T}}=\left\langle\mathrm{Y}_{\mathrm{S}}, \mathcal{F}\right\rangle$. Furthermore, $\mathcal{T}_{\mathrm{T}}=\mathrm{X}_{\mathrm{T}} \cap \mathrm{H}_{\mathrm{S}}$.

We claim that $\mathcal{T}_{\mathrm{T}}=\mathcal{T}$. First observe that any $N \in \mathrm{H}_{\mathrm{S}}$ satisfies $N \in(\mathrm{T}[<-1])^{\perp}$ because $\mathrm{T} \subseteq \mathrm{S} * \mathrm{~S}[1]$. Therefore $N \in \mathrm{H}_{\mathrm{S}}$ lies in $\mathcal{T}_{\mathrm{T}}$ if and only if $N \in(\mathrm{T}[-1])^{\perp}$. By Lemma 2.8, $N \in \operatorname{Fac}(M)$ if and only if $\operatorname{Hom}_{\mathrm{H}_{\mathrm{s}}}(\widetilde{\sigma}, N)$ is surjective, where we use the notation of Remark 2.11. By Remark 2.11, we can lift $\widetilde{\sigma}$ to the functor category as $\left.\left.\operatorname{Hom}_{\mathrm{D}}(-, \widetilde{S})\right|_{\mathrm{S}} \stackrel{(-, f)}{\rightarrow} \operatorname{Hom}_{\mathrm{D}}\left(-, S_{0}\right)\right|_{\mathrm{s}}$, and note that via the restricted Yoneda functor (e.g. [22, Remark 3.1]), $\operatorname{Hom}_{\mathrm{H}_{\mathrm{s}}}(\widetilde{\sigma}, N)$ is surjective if and only if

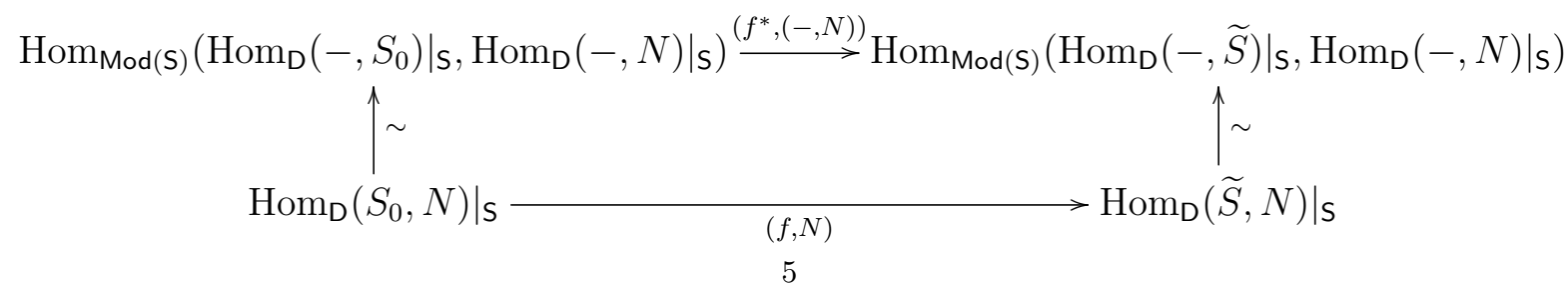


is surjective, where the vertical arrows are given by the Yoneda embedding. But since an additive generator of $\mathrm{T}$ is given as the mapping cone

$$
\widetilde{S} \stackrel{f}{\rightarrow} S_{0} \rightarrow T \rightarrow \widetilde{S}[1]
$$

and $\left.\operatorname{Hom}_{\mathrm{D}}\left(S_{0}[-1], N\right)\right|_{\mathrm{S}}=0$ since $N \in \mathrm{H}_{\mathrm{S}}$, we have $N \in(\mathrm{T}[-1])^{\perp}$ if and only if $N \in \operatorname{Fac}(M)$. Hence $\mathcal{T}_{\mathrm{T}}=\mathcal{T}$. It follows that $(\mathrm{X}, \mathrm{Y})=\left(\mathrm{X}_{\mathrm{T}}, \mathrm{Y}_{\mathrm{T}}\right)$, i.e. any t-structure intermediate with respect to $\left(\mathrm{X}_{\mathrm{S}}, \mathrm{Y}_{\mathrm{S}}\right)$ is algebraic.

In order to prove the key lemma, Lemma 3.5, we shall need two auxiliary results. The first one is the following straightforward observation; cf. [32, Lemma 2.9].

Lemma 3.2. Suppose $(\mathrm{X}, \mathrm{Y})$ is a bounded t-structure on $\mathrm{D}^{b}(\Lambda)$ and $\left(\mathrm{X}_{\mathrm{S}}, \mathrm{Y}_{\mathrm{S}}\right)$ is an algebraic t-structure on $\mathrm{D}^{b}(\Lambda)$. There exist integers $m \geq n$ such that $\mathrm{X}_{\mathbf{S}}[m] \subseteq \mathbf{X} \subseteq \mathbf{X}_{\mathbf{S}}[n]$.

Proof. Note that $\mathbf{X}_{\mathbf{S}}[m] \subseteq \mathbf{X} \subseteq \mathbf{X}_{\mathbf{S}}[n]$ is equivalent to $\mathbf{Y}_{\mathbf{S}}[m] \supseteq \mathbf{Y} \supseteq \mathbf{Y}_{\mathbf{S}}[n]$. Since $\left(\mathrm{X}_{\mathrm{S}}, \mathrm{Y}_{\mathrm{S}}\right)$ is algebraic, there exist finitely many simple objects $X_{1}, \ldots, X_{t} \in \mathrm{H}_{\mathrm{S}}$ such that $\left\langle X_{1}, \ldots, X_{t}\right\rangle=\mathrm{H}_{\mathrm{S}}$. The boundedness of $(\mathrm{X}, \mathrm{Y})$ asserts the existence of an integer $k$ such that $X_{i} \in \mathrm{X}[k]$ for each $1 \leq i \leq t$, whence $\mathrm{H}_{\mathrm{S}}[\geq 0] \subseteq \mathrm{X}[k]$. Thus, $\left(\mathrm{H}_{\mathrm{S}}[\geq 0]\right)^{\perp}=\mathrm{Y}_{\mathrm{S}} \supseteq \mathrm{Y}[k]$, and we can take $m=-k$. Analogously, there also exists an $l$ such that $X_{i} \in \mathrm{Y}[l]$ for each $1 \leq i \leq t$, so that $\mathrm{H}_{\mathrm{S}}[<0] \in \mathrm{Y}[l-1]$ and ${ }^{\perp}\left(\mathrm{H}_{\mathrm{S}}[<0]\right)=\mathrm{X}_{\mathrm{S}} \supseteq \mathrm{X}[l-1]$, and we can take $n=1-l$.

Our second auxiliary result is the following lifting and restriction lemma.

Lemma 3.3. If $(\mathrm{X}, \mathrm{Y})$ is a t-structure on $\mathrm{D}^{b}(\Lambda)$, then $(\widetilde{\mathrm{X}}, \widetilde{\mathrm{Y}}):=\left({ }^{\perp}\left(\mathrm{X}^{\perp}\right), \mathrm{X}^{\perp}\right)$ is a $t$ structure on $\mathrm{D}(\Lambda)$ such that $\left(\widetilde{\mathrm{X}} \cap \mathrm{D}^{b}(\Lambda), \widetilde{\mathrm{Y}} \cap \mathrm{D}^{b}(\Lambda)\right)=(\mathrm{X}, \mathrm{Y})$. Moreover, if there are integers $m \geq n$ such that $\mathbf{X}_{\mathbf{S}}[m] \subseteq \mathbf{X} \subseteq \mathbf{X}_{\mathbf{S}}[n]$ for some algebraic t-structure $\left(\mathbf{X}_{\mathbf{S}}, \mathbf{Y}_{\mathbf{S}}\right)$, then we also have $\widetilde{\mathrm{X}_{\mathrm{S}}}[m] \subseteq \widetilde{\mathrm{X}} \subseteq \widetilde{\mathrm{X}_{\mathrm{S}}}[n]$.

Proof. Since $\mathrm{D}^{b}(\Lambda)$ is essentially small, [33, Corollary 3.5] says that $(\widetilde{\mathrm{X}}, \widetilde{\mathrm{Y}})$ is indeed a t-structure. Since $\operatorname{Hom}_{D(\Lambda)}(X, \widetilde{Y})=0$ by definition, the inclusion $X \subseteq \widetilde{X}$ holds. Since $\operatorname{Hom}_{\mathrm{D}(\Lambda)}(\mathrm{X}, \mathrm{Y})=0$ we also get $\mathrm{Y} \subseteq \widetilde{\mathrm{Y}}$. Thus, $\left(\widetilde{X} \cap \mathrm{D}^{b}(\Lambda), \widetilde{Y} \cap \mathrm{D}^{b}(\Lambda)\right)=(\mathrm{X}, \mathrm{Y})$. For the final statement, note that $\mathbf{X}_{\mathbf{S}}[m] \subseteq \mathbf{X} \subseteq \mathbf{X}_{\mathbf{S}}[n]$ if and only if $\left(\mathbf{X}_{\mathbf{S}}[m]\right)^{\perp} \supseteq \mathbf{X}^{\perp} \supseteq\left(\mathbf{X}_{\mathbf{S}}[n]\right)^{\perp}$, that is $\widetilde{Y_{\mathrm{S}}}[m] \supseteq \widetilde{\mathrm{Y}} \supseteq \widetilde{\mathrm{Y}_{\mathrm{S}}}[n]$.

Remark 3.4. We observe that the lift $\left(\widetilde{X_{S}}, \widetilde{Y_{S}}\right)$ of an algebraic t-structure $\left(X_{S}, Y_{S}\right)$ on $\mathrm{D}^{b}(\Lambda)$ is a silting t-structure on $\mathrm{D}(\Lambda)$ in the sense of [7]. Namely, let $\mathrm{U}=(\mathrm{S}[<0])^{\perp}$ and $\mathrm{V}=(\mathrm{S}[\geq 0])^{\perp}$, where the orthogonals are taken in $\mathrm{D}(\Lambda)$. We claim that $(\mathrm{U}, \mathrm{V})=\left(\widetilde{\mathrm{X}_{\mathrm{S}}}, \widetilde{\mathrm{Y}_{\mathrm{S}}}\right)$. Since $S$ is a silting subcategory we have $S[\geq 0] \subseteq X_{S}$. Thus, $(S[\geq 0])^{\perp} \supseteq X_{S}{ }^{\perp}$, i.e. $\widetilde{Y_{S}} \subseteq \mathrm{V}$. For the reverse inclusion, observe that $\mathrm{X}_{\mathrm{S}} \subseteq \mathrm{U}$, so that $\widetilde{\mathrm{Y}_{\mathrm{S}}}=\mathrm{X}_{\mathrm{S}}{ }^{\perp} \supseteq \mathrm{U}^{\perp}=\mathrm{V}$.

We are now ready for the key lemma.

Lemma 3.5. Let $\Lambda$ be a silting-discrete finite-dimensional algebra. Suppose $(\mathrm{X}, \mathrm{Y})$ is a bounded t-structure on $\mathrm{D}^{b}(\Lambda)$. Then there exists a silting subcategory $\mathrm{S}=\operatorname{add}(S)$ and an algebraic t-structure $\left(\mathrm{X}_{\mathrm{S}}, \mathrm{Y}_{\mathrm{S}}\right)$ such that $\mathrm{X}_{\mathrm{S}}[1] \subseteq \mathrm{X} \subseteq \mathrm{X}_{\mathrm{S}}$.

Proof. Without loss of generality, we may assume $\mathbf{X}_{\mathrm{T}}[m] \subseteq \mathrm{X} \subseteq \mathrm{X}_{\mathrm{T}}$ for some algebraic t-structure $\left(\mathrm{X}_{\mathrm{T}}, \mathrm{Y}_{\mathrm{T}}\right)$. By Lemma 3.3, we can lift the t-structures and inclusions to $\mathrm{D}(\Lambda)$; these t-structures restrict to the given t-structures on $\mathrm{D}^{b}(\Lambda)$ and are decorated with tildes.

Following [19, Section 2], we set $\widetilde{Y_{1}}=\widetilde{Y_{\mathrm{T}}}[1] \cap \widetilde{Y}$, which, by [13, 33], gives rise to a t-structure $\left(\widetilde{\mathrm{X}_{1}}, \widetilde{\mathrm{Y}_{1}}\right)$. It has the following properties: $\widetilde{\mathrm{Y}_{\mathrm{T}}}[1] \supseteq \widetilde{\mathrm{Y}_{1}} \supseteq \widetilde{\mathrm{Y}_{\mathrm{T}}}$ (equivalently, 
$\left.\widetilde{\mathrm{X}_{\mathrm{T}}}[1] \subseteq \widetilde{\mathrm{X}_{1}} \subseteq \widetilde{\mathrm{X}_{\mathrm{T}}}\right)$; by [19, Lemma 2.12] we have $\widetilde{\mathrm{Y}_{1}}[m-1] \supseteq \widetilde{\mathrm{Y}} \supseteq \widetilde{\mathrm{Y}_{1}}$, i.e. $\widetilde{\mathrm{X}_{1}}[m-1] \subseteq$ $\widetilde{\mathrm{X}} \subseteq \widetilde{\mathrm{X}_{1}}$.

Now, by Proposition 2.3, there exists a torsion pair $(\widetilde{\mathcal{T}}, \widetilde{\mathcal{F}})$ on $\widetilde{\mathrm{H}_{\mathrm{T}}}$ such that $\widetilde{\mathrm{X}_{1}}=$ $\left\langle\widetilde{\mathcal{T}}, \widetilde{\mathrm{X}_{\mathrm{T}}}[1]\right\rangle$ and $\widetilde{\mathrm{Y}_{1}}=\left\langle\widetilde{\left.\mathrm{Y}_{\mathrm{S}}, \widetilde{\mathcal{F}}\right\rangle}\right.$. By [27, Corollary 3] or [30, Corollary 4.7], $\mathrm{H}_{\mathrm{T}} \simeq \operatorname{Mod}(\Gamma)$, where $\Gamma=\operatorname{End}(T)$. Since any torsion pair on $\operatorname{Mod}(\Gamma)$ restricts to a torsion pair on $\bmod (\Gamma)$, the t-structure $\left(\widetilde{X_{1}}, \widetilde{Y_{1}}\right)$ restricts to a t-structure $\left(\mathrm{X}_{1}, \mathrm{Y}_{1}\right)$ on $\mathrm{D}^{b}(\Lambda)$ such that $\mathrm{X}_{\mathrm{T}}[1] \subseteq \mathrm{X}_{1} \subseteq$ $\mathbf{X}_{\mathrm{T}}$. By Proposition 3.1. $\left(\mathbf{X}_{1}, \mathrm{Y}_{1}\right)$ is an algebraic t-structure with $\mathbf{X}_{1}[m-1] \subseteq \mathbf{X} \subseteq \mathbf{X}_{1}$. The lemma now follows by induction.

Proof of Theorem $A$. Let $(\mathrm{X}, \mathrm{Y})$ be a bounded t-structure in $\mathrm{D}^{b}(\Lambda)$ for a silting-discrete finite-dimensional algebra $\Lambda$. By Lemma 3.5, $(\mathrm{X}, \mathrm{Y})$ is intermediate with respect to an algebraic t-structure $\left(\mathrm{X}_{\mathrm{S}}, \mathrm{Y}_{\mathrm{S}}\right)$. By Proposition 3.1, $(\mathrm{X}, \mathrm{Y})$ is therefore algebraic.

\section{Stability CONDITIONS}

4.1. Stability conditions. Rather than give a formal definition of stability conditions, we give an equivalent formulation; see [12]. Let $\mathbb{H}=\{r \exp (i \pi \varphi) \mid r>0$ and $0<\varphi \leq$ 1\}. A stability function on an abelian category $\mathrm{H}$ consists of a group homomorphism $Z: K_{0}(\mathrm{H}) \rightarrow \mathbb{C}$ such that $Z(H) \in \mathbb{H}$ for each $H \in \mathrm{H}$. If $\mathrm{H}$ is a length category then a stability function is uniquely determined by its action on the simple objects.

Proposition 4.1 ([12, Proposition 5.3]). Specifying a stability condition $\sigma=(Z, \mathrm{H})$ on a triangulated category $\mathrm{D}$ is equivalent to specifying a bounded $t$-structure on $\mathrm{D}$ together with a stability function $Z: K_{0}(\mathrm{H}) \rightarrow \mathbb{C}$ on its heart $\mathrm{H}$ that satisfies the Harder-Narasimhan (HN) property.

Since any stability function on a length heart satisfies the HN property and, by Theorem A, all the bounded t-structures in $\mathrm{D}^{b}(\Lambda)$ are algebraic when $\Lambda$ is silting-discrete, we refrain from defining the HN property and refer the reader to [12]. From now on, since a bounded t-structure is determined by its heart we shall identify it with its heart.

Definition 4.2 ([12, Theorem 1.2]). Let D be a triangulated category. The set stab(D) of stability conditions $\sigma=(Z, \mathrm{H})$ forms a complex manifold called the stability manifold of D.

The set of stability conditions $\sigma=(Z, \mathrm{H})$ for some fixed $\mathrm{H}$ identifies a 'chamber' $\mathrm{C}_{\mathrm{H}}$ of $\operatorname{stab}(\mathrm{D})$. If $\mathrm{H}$ is algebraic, then $\mathrm{C}_{\mathrm{H}} \cong \mathbb{H}^{t}$, where $t$ is the number of nonisomorphic simple objects of $\mathrm{H}$; see [34].

4.2. Finite-type components. In [32] the tilting poset of a triangulated category D, denoted tilt(D), consists of bounded t-structures in $\mathrm{D}$ with the following partial order,

$(\mathrm{X}, \mathrm{Y}) \leq\left(\mathrm{X}^{\prime}, \mathrm{Y}^{\prime}\right) \Longleftrightarrow\left(\mathrm{X}^{\prime}, \mathrm{Y}^{\prime}\right)$ is obtained from $(\mathrm{X}, \mathrm{Y})$ by finitely many left HRS-tilts.

The subposet tilt $\mathrm{alg}_{\mathrm{al}}(\mathrm{D})$ consists of algebraic t-structures, where $(\mathrm{X}, \mathrm{Y}) \leq\left(\mathrm{X}^{\prime}, \mathrm{Y}^{\prime}\right)$ if and only if $\left(\mathrm{X}^{\prime}, \mathrm{Y}^{\prime}\right)$ is obtained from $(\mathrm{X}, \mathrm{Y})$ through finitely many left HRS-tilts, where each intermediate t-structure in the sequence of tilts is again algebraic. A component tilt $^{\circ}(\mathrm{D})$ of the tilting poset has finite type if each t-structure in tilt ${ }^{\circ}(\mathrm{D})$ is algebraic and has only finitely many torsion pairs in its heart. The following proposition is an immediate consequence of Theorem $\mathrm{A}$ and Theorem 2.6.

Proposition 4.3. Let $\Lambda$ be a silting-discrete finite-dimensional algebra. Then $\operatorname{tilt}\left(\mathrm{D}^{b}(\Lambda)\right)=$ tilt $\mathrm{talg}_{\mathrm{g}}\left(\mathrm{D}^{b}(\Lambda)\right)$ and each component $\operatorname{tilt}^{\circ}\left(\mathrm{D}^{b}(\Lambda)\right)$ of $\operatorname{tilt}\left(\mathrm{D}^{b}(\Lambda)\right)$ has finite type. 
By [32, Lemma 4.3], if tilt $^{\circ}(\mathrm{D})$ is a finite-type component of tilt(D) then

$$
\operatorname{stab}^{\circ}(\mathrm{D})=\bigcup_{\mathrm{H} \in \mathrm{tilt}^{\circ}(\mathrm{D})} \mathrm{C}_{\mathrm{H}}
$$

is a component of stab(D) called a finite-type component. The main theorem of [32] is the following.

Theorem 4.4 ([32, Theorem 4.9]). If $\operatorname{stab}^{\circ}(\mathrm{D})$ is a finite-type component of $\operatorname{stab}(\mathrm{D})$ then $\operatorname{stab}^{\circ}(\mathrm{D})$ is contractible.

4.3. Connectedness of the silting poset. Recall that the Hasse diagram (or quiver) of a poset $(S, \leq)$ has as vertices the elements of $S$, and there is an arrow $s \rightarrow t$ in the diagram if and only if $s<t$ and there is no element $x \in S$ such that $s<x<t$. A poset is called connected if its Hasse quiver is connected.

By [25, Theorem 7.13] and [5, Theorem 2.35], there is an arrow $(X, Y) \rightarrow\left(X^{\prime}, Y^{\prime}\right)$ in the Hasse quiver of $\operatorname{tilt}\left(\mathrm{D}^{b}(\Lambda)\right)$ if and only if the second t-structure can be obtained from the first by HRS tilt with respect to a torsion pair in the heart generated by a simple object. These are called simple left tilts in 32. In particular, it follows that the poset tilt $\mathrm{alg}_{\mathrm{a}}\left(\mathrm{D}^{b}(\Lambda)\right)$ and the silting poset $\mathbb{P}_{1}\left(\mathrm{~K}^{b}(\operatorname{proj}(\Lambda))\right)$ from Section 2.2 have isomorphic Hasse quivers.

Recall from [14 that a silting pair $\left(\mathrm{M}, \mathrm{M}^{\prime}\right)$ consists of a silting subcategory $\mathrm{M}$ of a triangulated category $D$ and a functorially finite subcategory $M^{\prime} \subseteq M$. The poset of silting pairs $\mathbb{P}_{2}(\mathrm{D})$ was defined in [14] via the opposite of the following partial order:

$$
\left(\mathrm{N}, \mathrm{N}^{\prime}\right) \geq\left(\mathrm{M}, \mathrm{M}^{\prime}\right) \stackrel{\text { def }}{\Longleftrightarrow} \mathrm{R}_{\mathrm{M}^{\prime}}(\mathrm{M}) \geq \mathrm{R}_{\mathrm{N}^{\prime}}(\mathrm{N}) \geq \mathrm{N} \geq \mathrm{M},
$$

where on the right-hand side the partial order is that of $\mathbb{P}_{1}(\mathrm{D})$ defined in Section 2.2 and $R_{M^{\prime}}(M)$ is the right mutation of $M$ at $M^{\prime}$; see [5] and [14, Section 5] for details. One gets the following theorem by observing that the proof in [14] works in this level of generality.

Theorem 4.5 ([14, Corollary 6.2 and Theorem 7.1]). Suppose $\Lambda$ is a silting-discrete finite-dimensional algebra. Then $\mathbb{P}_{2}\left(\mathrm{~K}^{b}(\operatorname{proj}(\Lambda))\right)$ is a $C W$ poset and $B \mathbb{P}_{2}\left(\mathrm{~K}^{b}(\operatorname{proj}(\Lambda))\right)$, the classifying space of the poset, is contractible.

Corollary 4.6. If $\Lambda$ is a silting-discrete finite-dimensional $\mathbf{k}$-algebra then tilt $_{\mathrm{alg}}\left(\mathrm{D}^{b}(\Lambda)\right)=$ $\operatorname{tilt}\left(\mathrm{D}^{b}(\Lambda)\right)$ is connected.

Proof. By Proposition 4.3, we have that tilt $\mathrm{alg}_{(\mathrm{D}}\left(\mathrm{D}^{b}(\Lambda)\right)=\operatorname{tilt}\left(\mathrm{D}^{b}(\Lambda)\right)$, and we only need to check that

$$
\operatorname{tilt}_{\mathrm{alg}}\left(\mathrm{D}^{b}(\Lambda)\right) \cong \mathbb{P}_{1}\left(\mathrm{~K}^{b}(\operatorname{proj}(\Lambda))\right)
$$

is connected. This is a generalisation of [14, Corollary 7.2]: the Hasse quiver of $\mathbb{P}_{1}\left(\mathrm{~K}^{b}(\operatorname{proj}(\Lambda))\right)$ is the 1-skeleton of $B \mathbb{P}_{2}\left(\mathrm{~K}^{b}(\operatorname{proj}(\Lambda))\right)$, which is contractible by Theorem 4.5, whence $\mathbb{P}_{1}\left(\mathrm{~K}^{b}(\operatorname{proj}(\Lambda))\right)$ is connected.

Remark 4.7. We prove Corollary 4.6 using Theorem 4.5 above because we believe the stronger statement that $B \mathbb{P}_{2}\left(\mathrm{~K}^{b}(\operatorname{proj}(\Lambda))\right)$ is contractible for any silting-discrete algebra is of independent interest. However, there is a direct argument, which we give below.

Given any $T \in \mathbb{P}_{1}\left(\mathrm{~K}^{b}(\operatorname{proj}(\Lambda))\right)$ we have $\Lambda[m] \leq T \leq \Lambda[n]$, for some integers $m \geq$ $n$. Silting discreteness together with [5, Proposition 2.36] give that $\Lambda[\mathrm{m}]$ can be obtained from $T$ by a finite number of irreducible left mutations. Therefore, each $T \in$ $\mathbb{P}_{1}\left(\mathrm{~K}^{b}(\operatorname{proj}(\Lambda))\right)$ is connected to some shift $\Lambda[m]$ in the Hasse quiver of $\mathbb{P}_{1}\left(\mathrm{~K}^{b}(\operatorname{proj}(\Lambda))\right)$. But, applying this argument with $T=\Lambda[m-1]$, we get that $\Lambda[m]$ and $\Lambda[m-1]$ are connected in that Hasse quiver. Therefore $\mathbb{P}_{1}\left(\mathrm{~K}^{b}(\operatorname{proj}(\Lambda))\right)$ is connected. 
Corollary B now follows directly from Corollary 4.6 by applying Theorem 4.4 .

Corollary 4.8. If $\Lambda$ is a silting-discrete finite-dimensional $\mathbf{k}$-algebra then the stability manifold $\operatorname{stab}\left(\mathrm{D}^{b}(\Lambda)\right)$ is contractible.

\section{REFERENCES}

[1] T. Adachi, T. Aihara and A. Chan, Tilting Brauer graph algebras I: classification of two-term tilting complexes, arXiv:1504.04827.

[2] T. Adachi, O. Iyama and I. Reiten, $\tau$-tilting theory, Compos. Math. 150 (2014), no. 3, 415-452.

[3] T. Adachi, Y. Mizuno and D. Yang, Silting-discreteness of triangulated categories and contractibility of stability spaces, arXiv:1708.08168.

[4] T. Aihara, Tilting-connected symmetric algebras, Algebr. Representation Theory 16 (2013), 873-894.

[5] T. Aihara and O. Iyama, Silting mutation in triangulated categories, J. London Math. Soc. 85 (2012), no. 3, 633-668.

[6] T. Aihara and Y. Mizuno, Classifying tilting complexes over preprojective algebras of Dynkin type, arXiv:1509.07387,

[7] L. Angeleri-Hügel, F. Marks and J. Vitória, Silting modules, Int. Math. Res. Not. IMRN (2016), no. 4, 1251-1284.

[8] I. Assem, D. Simson and A. Skowroński, "Elements of the representation theory of associative algebras, vol. I: Techniques of representation theory", London Mathematical Society Student Texts 65, Cambridge University Press, Cambridge, 2006.

[9] M. Auslander, Representation theory of Artin algebras I, Comm. Algebra 1 (1974), 177-268.

[10] A. Beilinson, J. Bernstein and P. Deligne, Faisceaux pervers, Astérisque 100, Soc. Math. France, Paris, 1982.

[11] A. Beligiannis and I. Reiten, Homological and homotopical aspects of torsion theories, Mem. Amer. Math. Soc. 188 (2007), no. 883.

[12] T. Bridgeland, Stability conditions on triangulated categories, Ann. of Math. 166 (2007), 317-345.

[13] N. Broomhead, D. Pauksztello and D. Ploog, Averaging t-structures and extension closure of aisles, J. Algebra 394 (2013), 51-78.

[14] N. Broomhead, D. Pauksztello and D. Ploog, Discrete derived categories II: the silting pairs CW complex and the stability manifold, J. London Math. Soc. 93 (2016), no. 2, 273-300.

[15] L. Demonet, O. Iyama and G. Jasso, $\tau$-tilting finite algebras, g-vectors and brick- $\tau$-rigid correspondence, arXiv: 1503.00285

[16] S. E. Dickson, A torsion theory for abelian categories, Trans. Amer. Math. Soc. 121 (1966), 223-235.

[17] G. Dimitrov, F. Haiden, L. Katzarkov and M. Kontsevich, Dynamical systems and categories in "The influence of Solomon Lefschetz in geometry and topology", vol. 621 of Contemp. Math., pp. 133-170, Amer. Math. Soc., Providence, RI, 2014.

[18] G. Dimitrov and L. Katzarkov. Bridgeland stability conditions on the acyclic triangular quiver, Adv. Math 288 (2016), 825-886.

[19] L. Fiorot, F. Mattiello and A. Tonolo, A classification theorem for t-structures, J. Algebra 465 (2016), 214-258.

[20] F. Haiden, L. Katzarkov and M. Kontsevich, Flat surfaces and stability structures, Publ. Math. Inst. Hautes Études Sci. 126 (2017), 247-318.

[21] D. Happel, I. Reiten and S. Smalø, Tilting in abelian categories and quasitilted algebras, Mem. Amer. Math. Soc. 120 (1996), no. 575, viii+ 88pp.

[22] O. Iyama, P. Jørgensen and D. Yang, Intermediate co-t-structures, two-term silting objects, $\tau$-tilting modules, and torsion classes, Algebra Number Theory 8 (2014), no. 10, 2413-2431.

[23] B. Keller, D. Vossieck, Aisles in derived categories, Bull. Soc. Math. Sér. A 40 (1988), no. 2, 239-253.

[24] A. King and Y. Qiu, Exchange graphs and Ext quivers, Adv. Math. 285 (2015), 1106-1154.

[25] S. Koenig and D. Yang, Silting objects, simple-minded collections, t-structures and co-t-structures for finite-dimensional algebras, Doc. Math 19 (2014), 403-438.

[26] E. Macrì, Stability conditions on curves, Math. Res. Lett. 14 (2007), no. 4, 657-672.

[27] P. Nicolás, M. Saorín and A. Zvonareva, Silting theory in triangulated categories with coproducts, arXiv: 1512.04700

[28] S. Okada, Stability manifold of $\mathbb{P}^{1}$, J. Algebraic Geom. 15 (2006), no. 3, 487-505.

[29] A. Polishchuk, Constant families of t-structures on derived categories of coherent sheaves, Mosc. Math. J. 7 (2007), no. 1, 109-134. 
[30] C. Psaroudakis and J. Vitória, Realisation functors in tilting theory, arXiv:1511.02677.

[31] Y. Qiu, Stability conditions and quantum dilogarithm identities for Dynkin quivers, Adv. Math. 269 (2015), 220-264.

[32] Y. Qiu and J. Woolf, Contractible stability spaces and faithful braid group actions, arXiv:1407.5986.

[33] M. Saorín and J. Stovicek, On exact categories and applications to triangulated adjoints and model structures, Adv. Math. 228 (2011), no. 2, 968-1007.

[34] J. Woolf, Stability conditions, torsion theories and tilting, J. London Math. Soc. 82 (2010), no. 3, 663-682.

Department of Mathematics and Statistics, Lancaster University, Lancaster, LA1 4YF, United KInGDOM

E-mail address: d.pauksztello@lancaster.ac.uk

Departamento de Matemáticas, Universidad de Murcia, Aptdo. 4021, 30100 Espinardo, Murcia, SPAIN.

E-mail address: msaorinc@um.es

Chebyshev Laboratory, St. Petersburg State University, 14th Line 29B, St. PetersBURG 199178, RUSSIA.

E-mail address: alexandrazvonareva@gmail.com 